\title{
William Hogarth in Göttingen: Wendungen eines Kultur- und Medientransfers
}

\author{
Christian Scholl
}

William Hogarth in Göttingen: die Wirkung der Arbeiten des zwischen den 1720er und 1760er Jahren in London tätigen Künstlers in der niedersächsischen Universitätsstadt der Aufklärungszeit stellt geradezu ein Paradebeispiel für Kommunikation und Kulturtransfer in der Personalunion dar. Aus Göttinger Sicht steht dabei selbstredend Georg Christoph Lichtenberg im Zentrum, der zwischen 1784 und 1785 seine Erklärungen „Ueber die Hogarthischen Kupferstiche" im „Göttinger Taschen Calender" sowie ab 1794 bis zu seinem Tod im Jahre 1799 die „Ausführlichen Erklärungen der Hogarthischen Kupferstiche“ publiziert hat. ${ }^{1}$ Indem Lichtenberg Texte zu Bildern verfasste, geht es bei dem hier zu verhandelnden Kulturtransfer zugleich um einen Medientransfer - und das mit allen Schwierigkeiten, die eine derartige Übertragung mit sich brachte. Das Thema ,Hogarth in Göttingen' geht aber noch darüber hinaus, denn die Rezeption dieses Künstlers fand im direkten Göttinger Umfeld Lichtenbergs auch innerhalb der bildkünstlerischen Medien statt: in den Nachstichen des Ernst Ludwig Riepenhausen. ${ }^{2}$ Dabei gibt es bei Riepenhausen verschiedene Modi der Hogarth-Rezeption, auf die im Folgenden noch einzugehen sein wird.

1 Wolfgang Promies (Hg.), Lichtenbergs Hogarth. Die Kalender-Erklärungen von Georg Christoph Lichtenberg mit den Nachstichen von Ernst Ludwig Riepenhausen zu den Kupferstich-Tafeln von William Hogarth, München/Wien 1999; Georg Christoph Lichtenberg, Ausführliche Erklärung der Hogarthischen Kupferstiche, mit verkleinerten aber vollständigen Copien derselben von E. Riepenhausen, 1.-5. Lieferung, Göttingen 1794-1799. Nach Lichtenbergs Tod folgten bis 1835 noch Lieferung 6-14, deren Text zu beträchtlichen Teilen von anderen Verfassern stammt - vgl. die Übersicht in: Ulrich Joost / Gerd Unverfehrt (Hg.), Hogarth und die Nachwelt. Von Lichtenberg bis Hrdlicka, Ausst.-Kat. Kunstsammlung der Universität Göttingen, 13. November-18. Dezember 1988, Göttingen 1988, S. 104f. Zu den späteren Verfassern gehört auch der Göttinger Kunsthistoriker Johann Dominicus Fiorillo - vgl. Claudia Schrapel, Johann Dominicus Fiorillo. Grundlagen zur wissenschaftsgeschichtlichen Beurteilung der „Geschichte der zeichnenden Künste in Deutschland und den vereinigten Niederlanden“, Hildesheim u. a. 2004, S. 429-449.

2 Vgl. hierzu Max Kunze, Zwischen Antike, Klassizismus und Romantik. Die Künstlerfamilie Riepenhausen. Ausst.-Kat. Winkelmann-Museum Stendal, 20. Mai-22. Juni 2001, Mainz 2001, S. 1-5 und 11-22. 
Zudem beginnt das Problem des Medientransfers bei Hogarth nicht erst mit der schriftlichen und bildlichen Rezeption. Vielmehr erweisen sich dessen Bilder selbst bei genauerer Betrachtung als intermedial. Es sind visuelle Gebilde, in denen Texte immer wieder eine integrative Rolle spielen. Für einen Transfer macht sie dies eher noch fragiler und erklärungsbedürftiger - und das in einer Zeit, die zunehmend sensibel für die Eigengesetzlichkeit von Texten und Bildern wurde. ${ }^{3}$ Insofern hat man es im Falle von Hogarth mit einem potenzierten Kultur- und Medientransfer zu tun, bei dem sich interkulturelle Übertragungsprobleme innerhalb des von der Personalunion abgesteckten politischen Raumes immer wieder mit medialen Übertragungsschwierigkeiten überkreuzen. Dies macht die entsprechenden Kunstwerke in ihrer Göttinger Rezeption so kompliziert, aber auch so interessant.

Um sich diesem komplizierten, glücklicherweise aber auch ausgesprochen unterhaltsamen Thema zu nähern, soll im Folgenden ein ausgewähltes Bild von Hogarth in seinem Weg durch die Medien verfolgt werden. Die Wahl fiel dabei ganz pragmatisch auf dasjenige Werk, von dem ein Stich vom Künstler selbst in der Kunstsammlung der Universität Göttingen vorhanden ist: „Strolling Actresses dressing in a barn“, übersetzt: Wander-Schauspielerinnen, die sich in einer Scheune ankleiden Abb. 1). ${ }^{4}$

Der querformatige, $428 \times 539 \mathrm{~mm}$ messende Stich zeigt das Innere einer Scheune. Balken und Dachschrägen suggerieren, dass der Blick tiefenräumlich in Richtung einer Giebelwand geht. Diese tiefer im Bildraum liegende Wand kann man jedoch nur im oberen Bereich des Bildes sehen, während im unteren Bereich bildparallel eine Bretterwand eingezogen ist, welche die Bühne für das figuren- und requisitenreiche Geschehen im Vorder- und Mittelgrund bietet. Dabei trifft die Bezeichnung ,Bühne' beinahe auch im wörtlichen Sinne zu, da es sich bei den dargestellten Personen um Schauspieler (bzw. überwiegend um Schauspielerinnen) handelt. Allerdings werden diese nicht bei der Aufführung eines Bühnenstücks gezeigt, sondern bei den geschäftigen Vorbereitungen, die dieser Aufführung vorausgehen. Hieraus ergeben sich eine Vielzahl von Brüchen zwischen der Höhenlage, die das Bühnenstück mit seinen Göttinnen und Heldinnen und den Requisiten einer möglichst perfekten Illusion einerseits beansprucht, und den desillusionierend profanen Tätigkeiten, denen die Akteure hier nachgehen. Ein erheblicher Teil des Witzes dieses Blattes besteht darin, dass Hogarth diesen Tätigkeiten selbst etwas Theatralisches verleiht, wie er ja die Schauspielerinnen bereits weitgehend kostümiert auftreten lässt. Insofern erscheint

3 Vgl. Moritz Wullen, Bild und Text. Hogarths Medienmix, in: Martina Dillmann / Claude Keisch (Hg.), Marriage a-la-mode. Hogarth und seine deutschen Bewunderer, Ausst.-Kat. Altes Museum Berlin, 18. Dezember 1998 - 28. Februar 1999, Städelsches Kunstinstitut und Städtische Galerie Frankfurt a. M., 25. März - 20. Juni 1999, Berlin 1998, S. 11-15.

4 William Hogarth, Strolling Actresses dressing in a barn, 1738, Radierung und Kupferstich, 428 x 539 mm, Kunstsammlung der Georg-August-Universität Göttingen. Vgl. auch Ronald Paulson, Hogarth's Graphic Works, New Haven / London 21970, Bd. 1, S. 182f.; Berthold Hinz / Hartmut Krug (Hg.), William Hogarth. Das vollständige druckgraphische Werk, Gießen ${ }^{3} 1988$, S. $73 f$. 


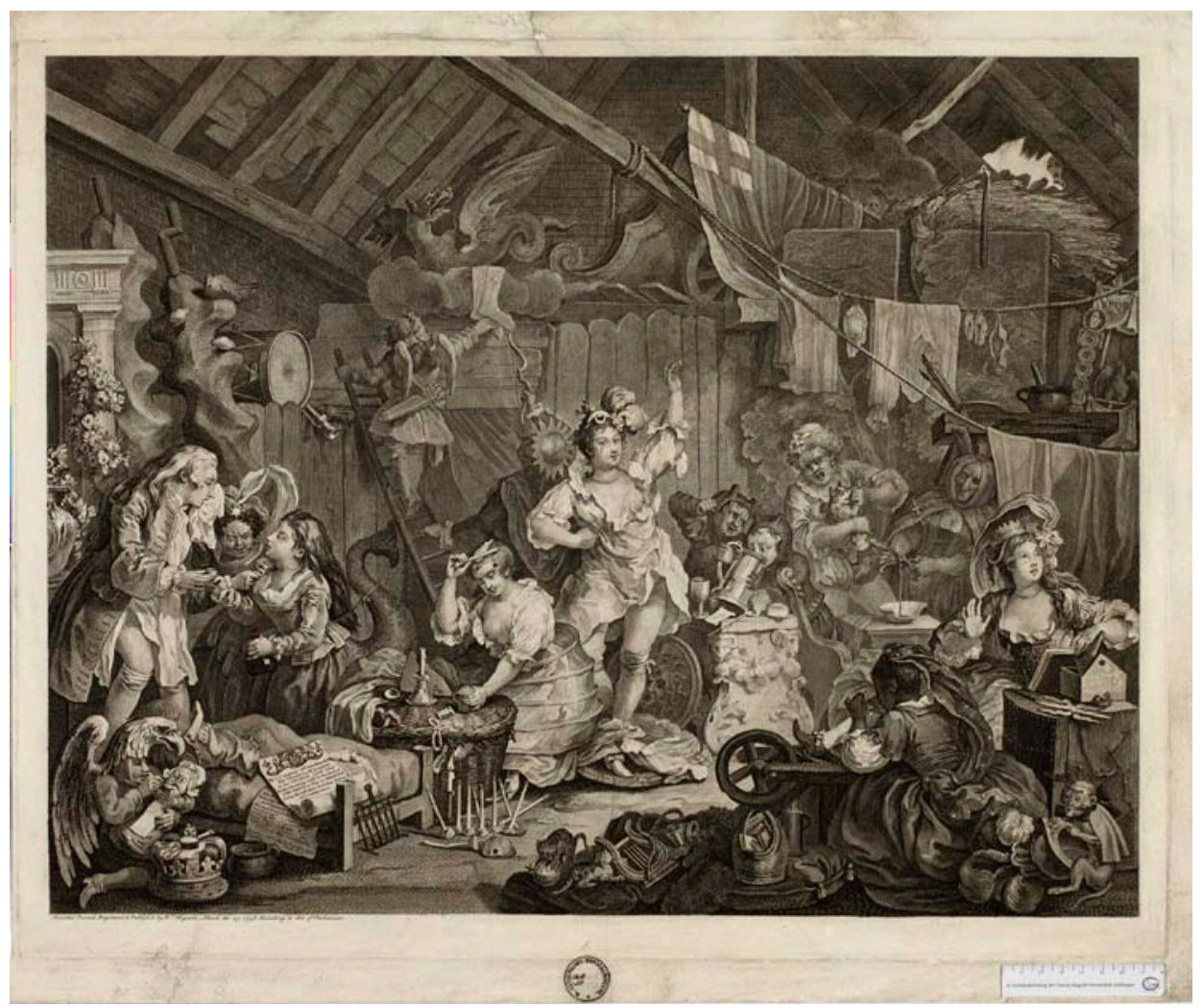

Abb. 1: William Hogarth: Strolling Actresses dressing in a barn, 1738, Radierung und Kupferstich, $428 \times 539$ mm;

Kunstsammlung der Georg-August-Universität Göttingen, Foto: Kristina Bohle

die formale Gestaltung des Raumes als Bühne - mit Kulissen, einem regelrechten Schnürboden und einer Art Hinterbühne - durchaus konsequent.

Das schauspielerhafte Agieren der Personen zeigt sich vor allem an der frontal dargestellten Figur im Zentrum des Blattes. Die Darstellerin der Mondgöttin Diana bewegt sich in ihrem derangierten Kleid in einer Art und Weise, die für Schauspieler und Tänzer der ersten Hälfte des 18. Jahrhunderts charakteristisch war. ${ }^{5}$ Links zu ihren Füßen talgt sich eine weitere Akteurin mit Krinoline das Haar, wobei sie einen Korb als Tisch benutzt. Direkt hinter ihr befindet sich - in Rückenansicht gezeigt -

5 Vgl. u. a. Margit Legler, Reinhold Kubik, Prinzipien und Quellen der barocken Gestik, in: Bert Siegmund (Hg.), Gestik und Affekt in der Musik des 17. und 18. Jahrhunderts. XXVIII. Internationale wissenschaftliche Arbeitstagung Michaelstein, 19. bis 21. Mai 2000, Michaelsteiner Konferenzberichte 63, Dössel 2003, S. 43-63. 
die Darstellerin einer Gottheit mit einer Sonne hinter dem Kopf. ${ }^{6}$ Sie gibt mit einem Bogen einem Kind, das als geflügelter Amor verkleidet ist, Anweisungen. Dieses ist auf eine Leiter hinaufgestiegen, um Strümpfe, die an einer Wolkenkulisse zum Trocknen angehängt sind, zu prüfen oder herunterzuholen. Darüber erkennt man auf dem Dachboden einen Drachenwagen, während links an die Bretterwand weitere Kulissenelemente gelehnt sind: Vor allem bewegliche Meereswellen, auf denen Hühner sitzen. Darunter tritt vom linken Bildrand her eine gestisch und mimisch leidend dargestellte Person ins Bild, der eine als Sirene mit Fischschwanz verkleidete Frau ein Getränk reicht. Eine ältere Darstellerin der Aurora ist derweilen damit beschäftigt, der Sirenen-Darstellerin an der Schulter einen Parasiten auszudrücken. In der linken unteren Ecke hockt eine als Adler verkleidete Frau, die ihr Kind füttert - der dazugehörige Breitopf ist auf einer Krone abgestellt.

Wendet man sich der rechten Bildhälfte zu, so sieht man links neben der Diana-Darstellerin zwei als Teufel verkleidete Kinder, die sich an einem antikisierenden Altar um einen Maßkrug balgen. Rechts daneben wird ein Tisch wirklich zum Opferaltar: Eine Frau hält eine Katze fest, der eine andere ein Stück vom Schwanz abschneidet, um daraus Theaterblut zu gewinnen. Davor schiebt sich in der rechten unteren Bildecke eine große Repoussoirgruppe. Ganz rechts arbeitet eine sitzende Schauspielerin offenbar daran, ihren Text zu repetieren. An ihren Beinen ist ein als Rückenfigur dargestelltes Mädchen damit beschäftigt, Strumplöcher zu schließen. Rechts davor hockt ein Affe mit einem Helm. Die dunkel gehaltene Repoussoirgruppe läuft etwa in der Bildmitte mit einem umgestürzten Karren, einer mit Büchern vollgestopften Bischofsmütze und zwei Katzen, die mit einem Reichsapfel und einer Lyra spielen, aus.

Ganz oben rechts sind auf dem Stich zwischen gut bestückten Wäscheleinen römische Heereszeichen zu entdecken. Eine Person schaut durch ein Loch in der Decke, während die Köpfe zweier weiterer Personen auf dem Dachboden hervorlugen.

\section{Von London nach Göttingen: Wendungen eines Medientransfers}

Die Umstände, unter denen die Blätter des in London geborenen und dort wirkenden Künstlers William Hogarth in die Hände des Göttinger Professors Georg Christoph Lichtenberg gelangten, sind von der Forschung bereits gut erschlossen worden. ${ }^{7} \mathrm{Zu}$

6 Die ikonographische Deutung wird frühzeitig diskutiert: Lichtenberg denkt in seinen Kalender-Erklärungen zunächst an Phöbus: Promies, 1999 (wie Anm. 1), S. 28. In der Ausführlichen Erklärung von 1794 deutet er sie unter Bezug auf weitere Quellen als Jupiter: Lichtenberg, 1794-1799 (wie Anm. 1), 1. Lieferung, S. 15.

7 Vgl. hierzu u. a. Ulrich Joost, Lichtenbergs Bilderklärungen, in: Joost/Unverfehrt, 1988 (wie Anm. 1), S. 14-22; Karl Arndt, „Orbis pictus“: Zu Lichtenbergs Hogarth-Erklärungen, in: Niederdeutsche Beiträge 
ihren Voraussetzungen gehört, dass Hogarth schon zuvor in Kreisen der Aufklärung kein Unbekannter war. Namentlich seine 1753 erschienene Schrift „The Analysis of beauty “ wurde in Deutschland intensiv diskutiert. ${ }^{8}$ Auch für Lichtenberg ist dementsprechend eine frühzeitige Kenntnis des Schaffens dieses Künstlers überliefert.

Entscheidend wurde für den Göttinger Gelehrten schließlich eine Reise, die ihn 1774 bis 1775 nach London führte. Dort erwarb Lichtenberg von der Witwe Hogarths, wie er selbst schreibt, zwey vollständige Exemplare der Werke, eines für einen Freund in Deutschland, und eines für mich. ${ }^{9}$ Damit sind die druckgraphischen Reproduktionen gemeint - die Gemälde scheint Lichtenberg nicht gesehen zu haben. ${ }^{10}$ Für das Thema ,Medientransfer' ist dieser Umstand überaus bedeutsam, denn nahezu alle Stiche von Hogarth gehen auf Gemälde desselben Künstlers zurück. Leider ist das entsprechende Bild für "Strolling Actresses dressing in a barn “ 1874 verbrannt. ${ }^{11}$ Blickt man aber auf andere Beispiele wie etwa die erhaltene Gemäldeserie zu „Marriage à la Mode“, so rückt ein Sachverhalt in den Fokus, der für Reproduktionsgraphik keineswegs ungewöhnlich ist: Die Stiche geben die Gemälde seitenverkehrt wieder. ${ }^{12}$ Mit einer solchen, gleichsam buchstäblichen Wendung im Medientransfer muss man auch bei den „Strolling Actresses" rechnen.

Mit dem Gemälde ist das künstlerisch vermutlich bedeutende Anfangsglied der medialen Kette von „Strolling Actresses“ für immer verloren. Rezeptionsgeschichtlich waren die Stiche freilich von vornherein wirksamer. Sie waren es auch, die Lichtenberg veranlassten, sich verstärkt mit dem künstlerischen Schaffen von Hogarth auseinanderzusetzen. Zurück in Göttingen, sammelte er Material zu den Blättern und entwickelte den Plan, Kommentare zu verfassen. Aus finanzieller Not sah er sich allerdings 1782 gezwungen, die Stiche an die Göttinger Universitätsbibliothek zu verkaufen. Immerhin erbat er sich, daß ich meinen Alten Freund zu weilen wieder auf meine Stube kommen lassen darf. ${ }^{13}$

zur Kunstgeschichte 33 (1994), S. 113-140; Ders., „....anschauende Kenntnis des Menschen in allen Ständen..." Georg Christoph Lichtenberg als Dolmetsch von William Hogarth, in: Dillmann/Keisch, 1999 (wie Anm. 3), S. 108-123; Hans-Georg von Arburg, Kunst-Wissenschaft um 1800. Studien zu Georg Christoph Lichtenbergs Hogarth-Kommentaren, Lichtenberg-Studien 11, Göttingen 1998, S. $13 \mathrm{f}$.

8 Vgl. Arndt, 1994 (wie Anm. 7), S. 114.

9 Promies, 1999 (wie Anm. 1), S. 157. Zu der Frage, welche Blätter in diesen „vollständigen Exemplare[n]“ enthalten waren, vgl. Arndt, 1994 (wie Anm. 7), S. 113f.

10 Vgl. Arndt, 1994 (wie Anm. 7), S. 108.

11 Vgl. Paulson, 1970 (wie Anm. 4), Bd. 1, S. 182.

12 Dies gilt für alle Stiche der Serie „Marriage à la mode“: vgl. Dillmann/Keisch, 1998 (wie Anm. 3), S. 52-65 mit Paulson, 1970 (wie Anm. 4), Bd. 2, Tafeln 268-273.

13 Georg Christoph Lichtenberg an Christian Gottlob Heyne, den 6. Mai 1782, in: Ulrich Joost/Albrecht Schöne (Hg.), Georg Christoph Lichtenberg. Briefwechsel. Bd. 2: 1780-1784, München 1985, S. 319 (Nr. 909). Vgl. auch Arndt, 1994 (wie Anm. 7), S. 115. 
Seit 1778 war Lichtenberg für den von Johann Christian Dieterich herausgegebenen „Göttinger Taschen Calender" tätig. Für diesen schrieb er ab dem Jahrgang 1784 regelmäßig „Ueber die Hogarthischen Kupferstiche“ - eine Serie, die bis 1795 fortgesetzt wurde. ${ }^{14}$ Der Text zu „Herumstreifende Comödianten, die sich in einer Scheune ankleiden“, erschien gleich im ersten Jahrgang. ${ }^{15}$ Das ist mediengeschichtlich insofern von Bedeutung, als dieser Beitrag noch nicht von druckgraphischen Reproduktionen zu Hogarth-Stichen begleitet wird, sondern als reine Text-Publikation publiziert wurde. In seiner Einleitung äußert sich Lichtenberg hierzu:

Der Verfasser gegenwärtigen Aufsatzes hofft, den Lesern des Calenders einen Dienst zu erzeigen [...] wenn er einige Züge aus den Werken dieses unsterblichen Mannes, die nicht jedem verständlich seyn mögten, hier aufsammelt und erklärt, er wird gröstentheils ohnehin nur solche wählen, die auch noch ohne die Abbildung dabey zu haben, unterhalten können. ${ }^{16}$

Auch wenn Lichtenberg am Ende seiner Einleitung erörtert, wo und zu welchem Preis man die entsprechenden Druckgraphiken erwerben könne, ${ }^{17}$ entwickelt er zunächst einmal eine bildlose Beschreibung. Man hat es also mit einem wirklichen Medientransfer zu tun, bei dem ein Text das absente Bild ersetzen soll. Umso bemerkenswerter ist, wie Lichtenberg diesen Transfer umsetzt.

Selbst wenn es ausgesprochen verlockend erscheint, Lichtenberg ausführlich zu zitieren, sei hier doch zu einem eher abstrakten Mittel gegriffen, um dessen Gang der Beschreibung nachzuvollziehen (Abb. 2): Anhand von Pfeilen lässt sich erkennen, wie sich Lichtenberg in seinem Text am Bild entlangschreibt. Dabei fällt auf, dass er tatsächlich am linken Bildrand beginnt und sich dann in Schleifen allmählich nach rechts vorarbeitet. Im Großen und Ganzen ,scannt` er das Bild gleichsam von links nach rechts ab. Bezeichnenderweise folgt er dabei der Leserichtung. Dass man dieses Bild auch ganz anders beschreiben kann, konnte oben mit einer eigenen Beschreibung demonstriert werden, die von der Hauptfigur im Zentrum der Komposition ausging. Bei Lichtenberg wird zudem nicht zuerst der Aktionsraum charakterisiert, sondern vielmehr das Bild von links nach rechts Objekt für Objekt durchbeschrieben. Erst in der rechten Bildhälfte verliert dieser Vorgang an Systematik. Dabei scheint der Sprung durch das Sujet motiviert: Lichtenberg gelangt nämlich von der Katze, welcher der Schwanz gekürzt wird, zu den beiden Katzen, die im Vordergrund mit

14 Promies, 1999 (wie Anm. 1). Vgl. Arndt, 1994 (wie Anm. 7), S. 115-118; Arburg, 1998 (wie Anm. 7), S. 1315.

15 Promies, 1999 (wie Anm. 1), S. 27-30.

16 Promies, 1999 (wie Anm. 1), S. 8.

17 Promies, 1999 (wie Anm. 1), S. 8. 


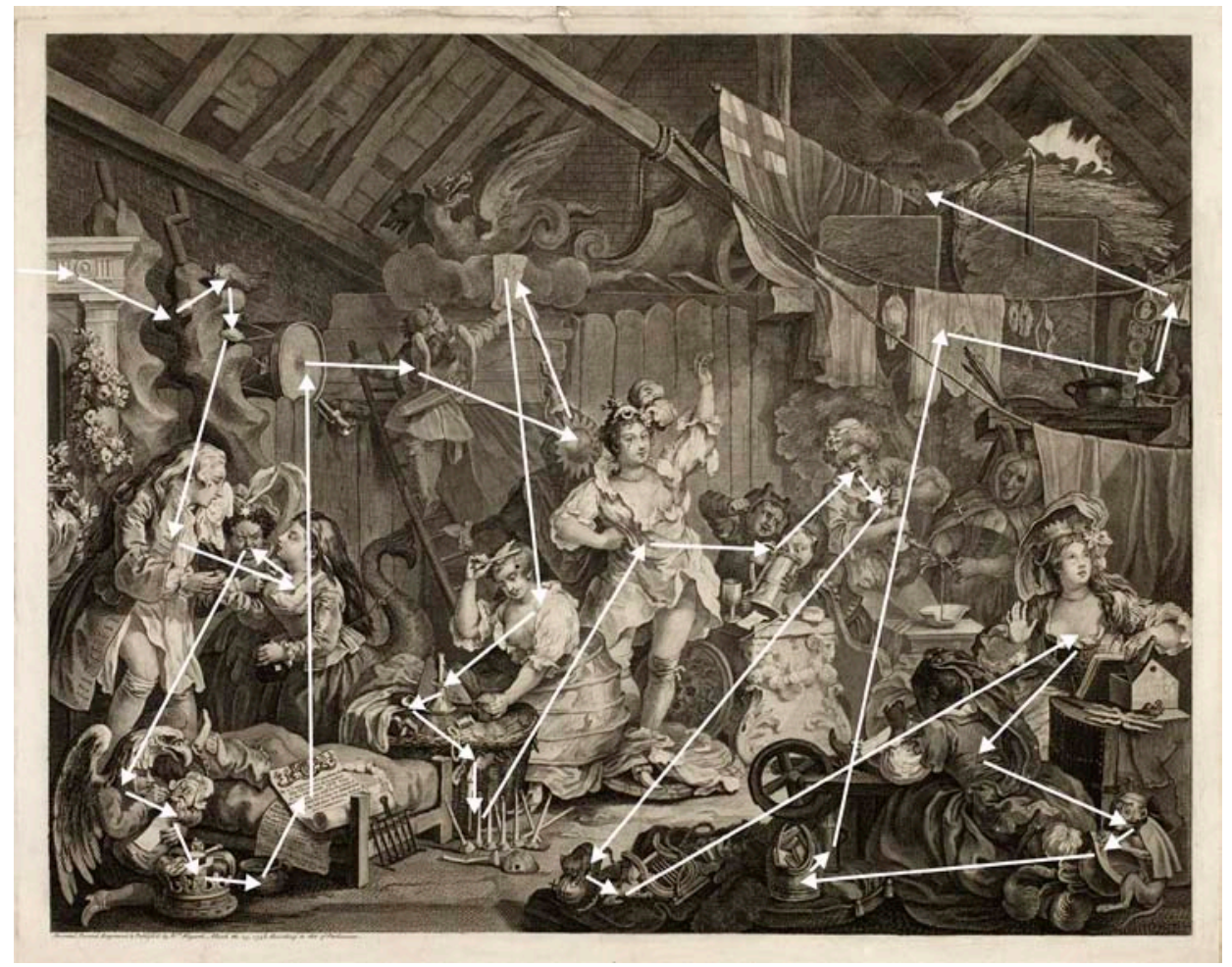

Abb. 2: William Hogarth: Strolling Actresses dressing in a barn mit graphischer Darstellung der Beschreibungsrichtung durch Georg Christoph Lichtenberg;

Kunstsammlung der Georg-August-Universität Göttingen, Foto: Kristina Bohle

Reichsapfel und Lyra spielen. ${ }^{18}$ In Folge dieses ,Katzensprungs' wird die bis dahin ausgesprochen kontinuierliche Bilderschließung zugunsten einer sich stärker zwischen den Ebenen hin- und herbewegenden Schilderung aufgegeben.

Sieht man sich bei den anderen Texten Lichtenbergs um, so erscheint das zumindest in der linken Bildhälfte relativ strikt durchgehaltene Beschreiben von links nach rechts eher als eine Besonderheit, die vermutlich mit der Fülle des auf diesem Blatt Dargestellten zusammenhängt. In diesem Falle wäre sie gerade kein Indiz für ein eher literarisches Vorgehen Lichtenbergs, sondern vielmehr eine Reaktion auf die spe-

18 Promies, 1999 (wie Anm. 1), S. 29: Neben dem Altar steht eine lautschreyende Frauensperson, die eine Katze hält, von der sie gebissen wird, weil man ihr die Spitze des Schwanzes abschneidet, um Blut fürs Trauerspiel zu erhalten; der übrige Stamm des Schwanzes bleibt stehen, für eine nächste Erndte, [...]. Ein junger Anflug von Katzen für den jungen Anflug von Trauerspielen, spielt fürs erste sein zeitiges Lustspiel unten vor der bisincta Diana; die eine mit einem Reichsapfel, und die andere lockt, oder ist im Begrif zu locken, Katzentöne aus der goldenen Leyer. 
zifische visuelle Erscheinung dieses konkreten Blattes. Tatsächlich ging der Verfasser ausgesprochen differenziert mit den Bildern Hogarths um.

Wie bereits erwähnt, war Lichtenbergs Bilderklärung im „Göttinger Taschen Calender“ von 1784 als Bildersatz gedacht. Ganz anders verhält es sich bei der „Ausführlichen Erklärung der Hogarthischen Kupferstiche", die in mehreren Lieferungen ab 1794 publiziert wurde. ${ }^{19}$ Diese erschien, wie der Untertitel deutlich macht, mit verkleinerten aber vollständigen Copien derselben von E. Riepenhausen. Damit ist der Göttinger Kupferstecher Ernst Ludwig Riepenhausen gemeint, der in den Jahrgän-

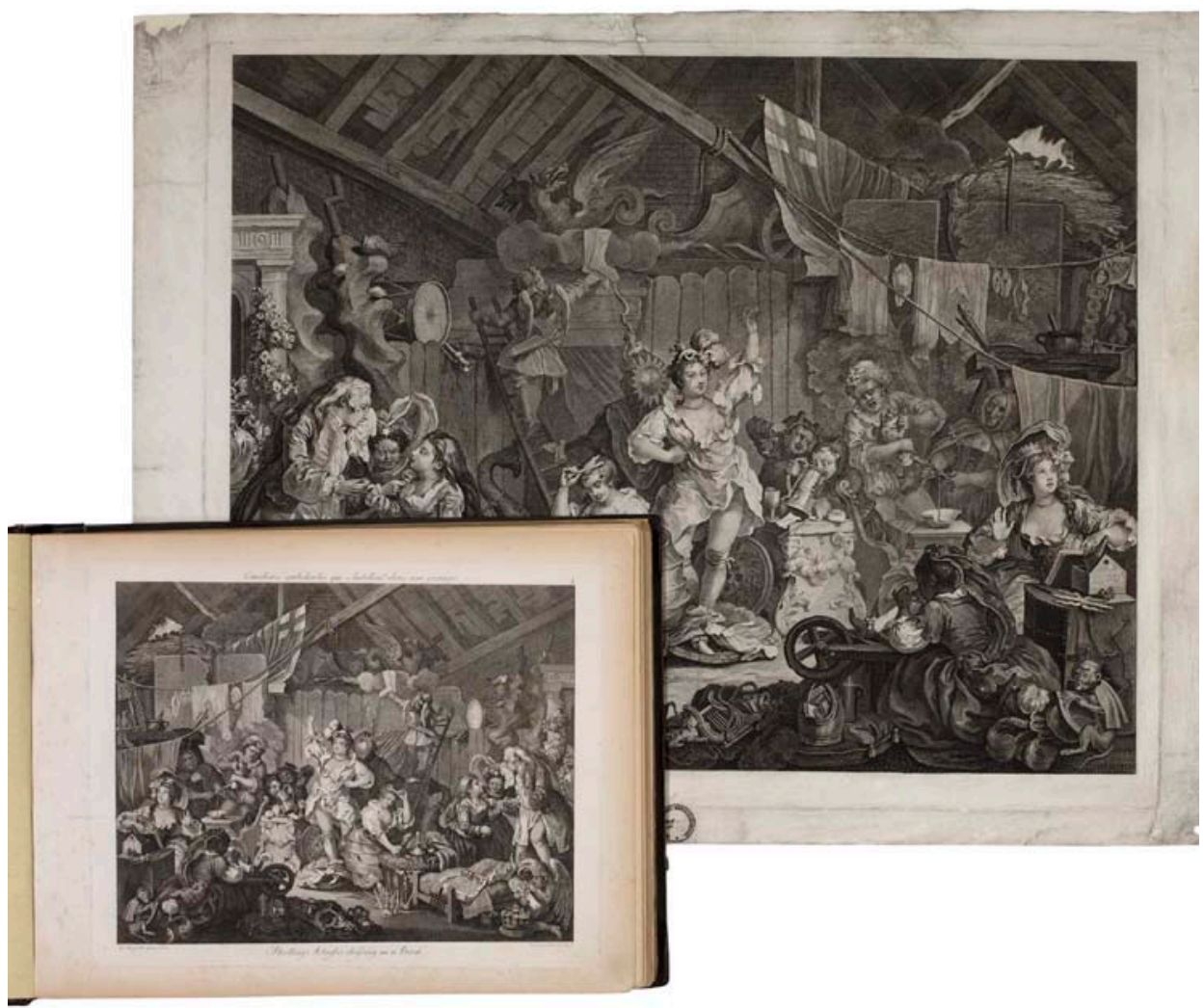

Abb. 3: Ernst Ludwig Riepenhausen nach William Hogarth: Strolling Actresses dressing in a barn, Kupferstich, 234 x 295 mm;

Kunstsammlung der Georg-August-Universität Göttingen, im Größenvergleich mit der Stichvorlage von Hogarth (wie Abb. 1), Foto: Kristina Bohle

19 Vgl. Joost, 1988 (wie Anm. 7), S. 16-19; Arburg, 1998 (wie Anm. 7), S. 15f.; Arndt, 1994 (wie Anm. 7), S. 118-121. 
gen ab 1785 für Lichtenberg bereits das Kupfer für den „Taschen Calender“ geliefert hatte. ${ }^{20}$ Das Bild „Herumstreichende Komödiantinnen“ ist das erste Werk überhaupt, das in der „Ausführlichen Erklärung “ besprochen wird. ${ }^{21}$

Vergleicht man Riepenhausens Nachstich mit Hogarths Originalstich, so fällt zunächst einmal der Größenunterschied ins Auge (Abb. 3). Da es vor allem darum ging, handlichere und sicher auch preisgünstigere Reproduktionen für das deutsche Publikum zu erstellen, sind die Riepenhausen-Drucke erheblich kleiner. Eine weitere augenfällige Differenz betrifft die Ausrichtung: Der Riepenhausen-Stich ist gegenüber der Hogarth-Vorlage wiederum seitenverkehrt. In diesem Fall hängt dies nicht damit zusammen, dass hier erneut einfach nur nach der Vorlage (hier: der Stichvorlage) gearbeitet wurde, die durch den Druck gespiegelt wurde. Gegen eine solche Erklärung sprechen andere Beispiele, bei denen Riepenhausen den Stich so angelegt hat, dass die Vorlage am Ende seitenrichtig wiedergegeben wurde. ${ }^{22}$ Vielmehr liegt hier eine bewusste Entscheidung vor, die Lichtenberg in seiner „Ausführlichen Erklärung“ auch entsprechend begründet hat:

Herr Riepenhausen hat Hogarth's Copie dieses Mal nicht umgezeichnet, und das, wie mich dünkt, mit sehr vielem Recht. Denn erstlich fällt nun das Licht wieder von der Linken ein, wie es sich gehört; zweitens schneidet nun die alte Frau an der Katze mit der rechten Hand, und drittens näht die Göttin der Nacht mit der Rechten. Hätte man auch annehmen wollen, Hogarth hätte bei der Alten seine Absichten gehabt, ihr die Scheere in die Linke zu geben; so war er doch gar der Mann nicht, der von einem so sehr mittelmäßigen Einfall zweimal auf einem und demselben Blatt Gebrauch hätte machen können, und viertens kommen nun Ganymed's Knopflöcher auch wieder an die linke Seite. Wollte man auch da sagen, der Rock wäre ein gewendeter. Lieber Himmel! Was für Unsinn ließe sich nicht durch eine solche Hermeneutik rechtfertigen! ${ }^{23}$

Mit der Existenz eines preiswerten, dem Publikum zugänglichen Nachstiches konnte Lichtenberg auch seine Beschreibungsstrategie ändern. Er stellte seine Schilderung jetzt unter die leitende Fragestellung, wie sich der Titel „Strolling Actresses“ mit den zum Teil maskulin wirkenden Figuren vereinen lässt, und ging generell auf sehr viel freiere Weise mit der Beschreibung des Blattes vor. Ganz offensichtlich ist

20 Zu Riepenhausens Stichen für die „Ausführliche Erklärung“ vgl. Ausst. Kat. Stendal, 2001 (wie Anm. 2), S. 11-22.

21 Lichtenberg, 1794-99 (wie Anm. 1), 1. Lieferung, 1794, S. 3-30.

22 Tatsächlich gibt der Großteil der Riepenhausen-Nachstiche die englischen Stiche von bzw. nach Hogarth seitenrichtig wieder. Umso mehr erscheint das seltene Spiegeln der Vorlagen bewusst eingesetzt worden zu sein. So sind bei den Vier Tageszeiten die Blätter „Morning“ und „Evening" gespiegelt, die übrigen hingegen nicht. Bei der Serie „A Rake’s Progres““ sind Blatt 3, 4 und 8 gespiegelt. Die Serie „Marriage à la Mode“ ist komplett gespiegelt, ebenso die Einzelblätter „Columbus Breaking the Egg“, „Scholars at a Lecture“, "Southwark Fair", „The Politician“ sowie „Before“ (aber nicht „After").

23 Lichtenberg, 1794-99 (Anm. 1), 1. Lieferung, 1794, S. 29. 
dies eine Beschreibung, die damit rechnet, dass die Leser das Blatt vor sich haben. ${ }^{24}$ So zeigt sich gerade im Vergleich der Beschreibungen die Sorgfalt und Umsicht, mit der Lichtenberg mit diesen Bildern umgeht. Man kann, um einen modernen Begriff zu wählen, geradezu von ,Medienkompetenz'sprechen.

Von eindrucksvoller Qualität sind im übrigen auch die Nachstiche von Lichtenbergs bildkünstlerischem Mitstreiter Riepenhausen. Bei einer Bewertung ist die unterschiedliche Bildgröße in Betracht zu ziehen, welche dem Stecher manche Feinheit verwehrt hat. Wie genau er unter diesen Bedingungen die Hogarthsche Vorlage übertragen hat, ist durchaus beachtlich. So darf man in diesem Falle wohl tatsächlich von einem gelungenen Medientransfer sprechen, der hier sowohl von Bild zu Text als auch von Bild zu Bild zwischen London und Göttingen vollzogen wird.

\section{Von England nach Deutschland: Aspekte eines Kulturtransfers}

Nun geht es bei der Übertragung der Bilder Hogarths von London nach Göttingen nicht allein um einen Medientransfer, sondern auch um das Überspringen kultureller Differenzen. Von Beginn an reflektiert Lichtenberg im „Göttinger Taschen Calender“ über die Möglichkeiten und Schwierigkeiten des von ihm betriebenen Kulturtransfers. Er tut dies ganz grundlegend, indem er die Kunst Hogarths zu charakterisieren sucht. Einerseits stilisiert er diesen dabei zu einem universal wirksamen Künstler. Andererseits muss er aber eingestehen, dass eine Vielzahl von Details in dessen Bildern vom Betrachter ein Zusatzwissen erfordert. So schreibt er im Vorwort „Ueber die Hogarthischen Kupferstiche“ über Hogarth:

Den allgemeinen Beyfall hat er, wie große Schriftsteller den ihrigen, der Darstellung allgemeiner menschlichen Natur, und der Sprache zu danken, die man in Lissabon so gut versteht als in Moskau. Allein ausser diesen Zeichen, die seinen

24 Thomas Althaus diagnostiziert bei Lichtenbergs „Ausführlicher Erklärung“ eine zunehmende Eigenwertigkeit des Textes gegenüber den Bildern. Er konstatiert, dass die Hogarth-Kommentare „mit ihren ausgestellten Worten beginnen, eigene Geschichte zu erzählen, gegen den Bezugshorizont, in den sie gestellt sind. Der wäre dann wie der Bezugshorizont jedes literarischen Textes, der etwa entstehungsgeschichtlich auszumachen ist, im letzten nichts weiter als die Veranlassung des Schreibens. Die Bewegung der Entfernung von ihrem Anlaß ist an diesen Texten genau zu beobachten; sie zeigen sich noch in der Arbeit daran, zeigen den Prozeß ihrer Metaphorisierung an den ausgestellten Worten vor und machen ihn sich so selbst zum Thema“. - Thomas Althaus, Das Uneigentliche ist das Eigentliche. Metaphorische Darstellung in der Prosa bei Lessing und Lichtenberg, Literatur als Sprache. Literaturtheorie - Interpretation - Sprachkritik 8, Münster 1991, S. 255. Abgesehen davon, dass diese Diagnose tendenziell überzogen ist - vgl. auch Arburg, 1998 (wie Anm. 7), S. 39 - macht ein Vergleich der jeweiligen Medienkonstellation bei der „Ausführlichen Erklärung“ und den Kalender-Erklärungen deutlich, dass sich Lichtenberg gerade da von der Bildvorlage löst, wo er diese nicht mehr „ersetzen“ und Ekphrasis leisten muss, indem diese nunmehr selbst als Nachstich verfügbar ist. Insofern kann die Lösung von der Vorlage gerade als Reaktion auf deren im Bild gesicherte Präsenz verstanden werden. 


\begin{abstract}
Werken die Verständlichkeit versichern, so lange als sie dauern werden, bedient er sich, um denselben einen Reiz für sein Zeitalter besonders zu geben, einer Menge anderer, die mit der Zeit verlöschen werden, und wohl zum Theil schon verloschen sind; auch selbst die allgemein verständlichen sind öfters so angebracht, daß sie nicht jeder gleich für wichtig hält, und also einen Gedanken entbehrt, den er sogleich würde gefunden haben, wenn er nur das Zeichen für wichtig gehalten hätte. ${ }^{25}$
\end{abstract}

Lichtenberg argumentiert auf der Basis aufklärerischer Semiotik, indem er zwischen universal wirksamen und konventionellen, der Erklärung bedürftigen Zeichen unterscheidet. ${ }^{26}$ Anders als bei rigideren Kunsttheoretikern seiner Zeit, welche Werke der bildenden Kunst weitgehend auf den Gebrauch natürlicher Zeichen einschränken wollten, ${ }^{27}$ fällt sein Urteil allerdings ausgesprochen positiv aus. Die Folgen hieraus übernimmt er selbst als Schriftsteller: Lichtenberg erklärt Hogarth. Dabei hat ein erheblicher Teil seiner Erörterungen damit zu tun, dass der Künstler für ein britisches Publikum mit einem spezifischen Vorwissen gearbeitet hat, das eines Transfers bedarf. ${ }^{28}$

Was dies bedeutet, lässt sich anhand des Blattes „Herumstreifende Schauspielerinnen, die sich in einer Scheune ankleiden“" sehr gut erörtern. Tatsächlich gewinnt die hier gezeigte Szene nämlich erheblich an Aussagekraft und Schärfe, wenn man die vom Künstler selbst in das Bild integrierten Texte zu deuten weiß. In der linken unteren Ecke des Hogarth-Stiches (beim Riepenhausen-Stich ist es die rechte) sieht man neben der als Adler verkleideten Frau mit dem Kleinkind eine Krone. Auf dieser ist das Breipfännchen abgestellt, aus dem das Kind gefüttert wird. Zwischen Krone und Breipfanne ist ein Blatt abgelegt, auf dem zu lesen ist: „The Act against strolling Play-

25 Promies, 1999 (wie Anm. 1), S. 7.

26 Grundlage ist die etwa von Theoretikern wie Christian Wolff, Alexander Gottlieb Baumgarten, Johann Jacob Bodmer, Johann Jacob Breitinger, Moses Mendelssohn oder Gotthold Ephraim Lessing vorgenommene Differenz zwischen ,natürlichen' und ,willkürlichen' Zeichen - vgl. u. a. Bengt Algot Sørensen, Symbol und Symbolismus in den ästhetischen Theorien des 18. Jahrhunderts und der deutschen Romantik, Kopenhagen 1963, S. 32-40.

27 Die weitgehende Festlegung der Bildkünste auf den Gebrauch ,natürlicher ' Zeichen verbindet sich mit der Utopie einer unmittelbar anschaulich wirkenden Kunst und manifestiert sich im ausgehenden 18. Jahrhundert insbesondere in der Allegoriekritik - vgl. Sørensen, 1963 (wie Anm. 26), S. 33-40.

28 In seiner „Ausführlichen Erklärung der Hogarthischen Kupferstiche“ unterscheidet Lichtenberg zwischen einem prosaischen und einem poetischen Weg, Hogarths Werke zu erklären, und reklamiert für sich selbst den zweiten. Als gemeinsamen Nenner bezeichnet er jedoch die Vermittlung der erforderlichen Sachinformationen: Man sagte, was die Dinge bedeuten, und machte besonders auf solche aufmerksam, die jemand, der nicht mit dem Lande des Künstlers, oder auch nicht mit dessen Genie bekannt ist, entweder ganz übersehen, oder wenn er sie auch bemerkt hätte, doch nicht gehörig verstanden haben würde. (Lichtenberg, 1794-99 (wie Anm. 1), 1. Lieferung, 1794, S. IV). In diesem Zusammenhang verweist Lichtenberg mehrfach auf seinen häufigen Umgang mit Engländern (ebd., S. VIII, XIII). Tatsächlich baute er sich ein regelrechtes Netzwerk auf, um die Stiche interpretieren zu können - vgl. Arburg, 1998 (wie Anm. 7), S. 17. 
ers“. Dieser Hinweis führt zu einem konkreten historischen Dokument: 1737 wurde ein Gesetz erlassen, welches Theateraufführungen auf bestimmte Aufführungsstätten (die Theater Drury Lane, Covent Garden, die City von Westminster sowie den Aufenthaltsort des Königs) beschränkt. ${ }^{29}$ Daraus folgt, dass für die Schauspielertruppe, die Hogarth in seinem Stich beim Umkleiden in einer ländlichen Scheune zeigt, ein Existenzverlust unmittelbar bevorsteht. Der daneben auf dem Bett dargestelle Theaterzettel vertieft dies: By a Company of Comediens from the Theatres at London at the GEORGE INN This Present Evening will be Presented THE DEVIL to Pay in HEAVEN Being the last time of Acting Before the Act Commences. Es geht folglich um die letzte Vorstellung, welche diese Theatertruppe geben kann, bevor das Gesetz greift und derartige Vorstellungen verboten werden.

Von diesen schriftlich wiedergegebenen Sachinformationen hängt die Deutung des Bildes in nicht unerheblichem Maße ab. Dementsprechend geht auch Lichtenberg ausführlich hierauf ein. So schreibt er etwa in der „Ausführlichen Erklärung der Hogarthischen Kupferstiche" von 1794:

Bald wird es also vorbei seyn, gesetzt auch, daß sie, wie in Deutschland gewöhnlich ist, drei bis viermal hinter einander zum letzten Male spielen. Diese armen Teufel wird schwerlich etwas retten können, auch die feinste Sylbenstecherei englischer Rechtshändler nicht. Sagte die Acte, wie etwa im Deutschen, bloß against strolling actors (gegen herumstreichende Comödianten), so dürften sie nur sagen: wir streichen zwar, aber wir sind Comödiantinnen: so wären sie einstweilen so sicher in ihren Scheunen, als auf ihren Londonschen Theatern. So aber heißt es against strolling players, und gegen dieses Wort käme selbst eine Bande Hermaphroditen nicht auf. Aber wer weiß was sie dennoch thun. So wahr ist es überall, aber nirgends mehr als in England: um Recht zu thun in der Welt, braucht man nur sehr wenig zu wissen, allein um mit Sicherheit Unrecht thun zu können, muß man die Rechte studiren. ${ }^{30}$

Auffällig ist, wie Lichtenberg hier immer wieder zwischen Verweisen auf England und auf Deutschland hin- und herpendelt, während er gleichzeitig die Leitfrage nach dem Geschlecht der „Actresses“ weiterspinnt. Genau darin zeigt sich sein Bemühen um einen Kulturtransfer.

Die Konsequenz aus der ganz unmittelbaren Bedrohung der Schauspieler sieht er in einem anderen Bilddetail verwirklicht. Unter dem Korb mit der Aufschrift „Jewels“, an dem sich eine Schauspielerin die Haare talgt, befindet sich eine Kerze, deren Flamme bereits den Korb erfasst:

29 Vgl. Frederick Antal, Hogarth und seine Stellung in der europäischen Kunst, Dresden 1962, S. 90f.; Paulson, 1970 (wie Anm. 4), Bd. 1, S. 182; Ronald Paulson, Hogarth. His Life, Art, and Times, New Haven $/$ London 1971, Bd. 1, S. 395.

30 Lichtenberg, 1794-99 (wie Anm. 1), 1. Lieferung, 1794, S. 6. 
Ein brennendes Licht, das vermuthlich zum Anzünden der übrigen bereit steht, ist so nachlässig hingestellt, daß die Flamme desselben das Stroh, worein die Juwelen im Malter-Cassettchen gepackt sind, ergreift, und nicht allein die Juwelen (denn nach den neuesten Versuchen verfliegt der Demant im Feuer), sondern dieses ganze Pantheon mit allen seinen Herrlichkeiten aufliegen machen wird, wenn es die Göttinnen nicht bald gewahr werden. Also hier glimmt schon die Rechtskräftigkeit der Parlaments-Acte, von dem Schicksal selbst angefacht. ${ }^{31}$

So deckt Lichtenberg Bildstrategien auf, die für Hogarth ausgesprochen charakteristisch sind. Dabei gewinnt seine Erklär-Arbeit dadurch an Bedeutung, dass die vom Künstler angewandten Strategien im ausgehenden 18. Jahrhundert zunehmend in Verruf gerieten, indem das Ideal selbstevidenter Bilder propagiert wurde. ${ }^{32}$ Hogarths erklärungsbedürftige Medienkombination war insofern alles andere als unproblematisch.

\section{Von Kunstwerk zu Kunstwerk: Zur Kommunikation des künstlerischen Anspruchs}

Umso erstaunlicher erscheint es, wie Lichtenberg die künstlerische Gültigkeit von Hogarth beschwört. Dabei greift er gleich zu Beginn seiner Kalendererklärungen „Über die Hogarthischen Kupferstiche“ zum Höchsten, zu dem man als kunstverständiger Verfasser im ausgehenden 18. Jahrhundert überhaupt greifen kann - zu Raffael:

Es wird nicht leicht unter uns, oder überhaupt in der gesitteten Welt, einen Mann von Geist und Geschmack geben, der den Namen Hogarth nicht kennt. Auch ist der Mann in seiner Art gewißs so einzig, als Raphael in der seinigen, so verschieden auch die Wege seyn mögen, die beyde zu ihrem Ruhm betreten haben. ${ }^{33}$

31 Lichtenberg, 1794-99 (wie Anm. 1), 1. Lieferung, 1794, S. 12.

32 Man kann hier etwa auf Johann Wolfgang Goethes Weimarischen Kunstfreund Johann Heinrich Meyer verweisen, der in seinem Aufsatz „Über die Gegenstände der bildenden Kunst“ das Ideal eines sich selbst erklärenden Bildes propagiert hat - Johann Heinrich Meyer, Ueber die Gegenstände der bildenden Kunst, in: Propyläen. Eine periodische Schrifft 1,1 (1798), S. 20-54, hier S. 21: Man fordert von einem jeden Kunstwerke, daß es ein Ganzes für sich ausmache, und von einem Werke der bildenden Kunst besonders, daß es sich selbst ganz ausspreche. Es muß unabhängig seyn, die vorgestellte Handlung, der Gegenstand muß, im Wesentlichen, ohne äussere Beyhülfe, ohne Nebenerklärung, die man aus einem Dichter oder Geschichtschreiber schöpfen müßte, gefaßt und verstanden werden.

33 Promies, 1999 (wie Anm. 1), S. 7. 
Die Aussage, dass Hogarth in seiner Art so einzig sei als Raphael in der seinigen, erstaunt nicht zuletzt deshalb, weil Raffael in der Kunsttheorie dieser Zeit nicht als Spezialist galt, sondern als universaler Künstler. Noch mehr überrascht, dass Lichtenberg ausgerechnet einen Künstler wie Hogarth, dessen Werke klassische Idealvorstellungen immer wieder verletzen und nicht selten karikaturistisch überzeichnet erscheinen, mit Raffael in Beziehung setzt, dessen Kunst geradezu als Inbegriff des klassischen Ideals angesehen werden kann. ${ }^{34}$

Nun gehört es zu den besonders bemerkenswerten Facetten von Hogarths Schaffen, dass es selbst von einer intensiven Auseinandersetzung mit Werken und Bildtypen der Kunstgeschichte geprägt ist. ${ }^{35}$ Auch dies lässt sich am Beispiel des Bildes „Strolling Actresses dressing in an Barn" exemplarisch demonstrieren. Als künstlerische Referenz figuriert dabei tatsächlich kein Geringerer als Raffael selbst. Wie Frederick Antal plausibel machen konnte, lassen sich die beiden als Teufelchen verkleideten Kinder, die sich hinter einem antikisierenden Opferaltar um einen Bierkrug rangeln, als Zitate zweier Kinderfiguren in Raffaels Karton „Das Opfer zu Lystra“ deuten (Abb. 4). ${ }^{36}$ Hogarth hat die Kartons zu Wandteppichen in der Sixtinischen Kapelle in Rom, die zu den wertvollsten Kunstschätzen in England gehörten, zweifellos gekannt. Zu seinen Lebzeiten wurden sie (bis 1763) im Hampton Court Palace gezeigt. ${ }^{37}$ Dabei sind aus Raffaels lieblich dreinblickenden (und dreinfötenden) Ministranten bei Hogarth zwei ausgesprochen derbe Gesellen geworden, die ihre Teufelskostüme durchaus mit Leben erfüllen.

Die Art und Weise, wie sich Hogarth hier auf Raffael bezieht, hat also einen ausgesprochen parodistischen Charakter. Dies spricht gerade nicht für eine ernsthafte Berufung auf diesen Künstler, sondern eher für eine Distanzierung von dessen Idealismus. Man kann daraufhin sogar die Frage stellen, ob nicht sogar Lichtenbergs Einleitung selbst mit ihrem Loblied auf Hogarth als Distanzierung von dem idealistischen Kunstkonzept zu verstehen ist, wie man es um 1800 in Raffael verkörpert sah.

Tatsächlich ist der Sachverhalt aber wohl doch komplizierter - und dies sowohl bei Hogarth als auch bei Lichtenberg. So hat Hogarth ebenso versucht, sich als Maler ernster Historien in der Tradition Raffaels zu etablieren. ${ }^{38}$ Dieses - insgesamt eher er-

34 Zur Frage nach dem Karikaturenhaften von Hogarths Kunst und dessen Rezeption bei Lichtenberg vgl. Arburg, 1998 (wie Anm. 7) S. 182-194.

35 Vgl. hierzu insbesondere Werner Busch, Nachahmung als bürgerliches Kunstprinzip. Ikonographische Zitate bei Hogarth und in seiner Nachfolge, Studien zur Kunstgeschichte 7, Hildesheim/ New York 1977.

36 Antal, 1962 (wie Anm. 29), S. 90.

37 Vgl. John Shearman, Raphael's Cartoons in the Royal Collection of Her Majesty the Queen and the Tapestries for the Sistine Chapel, London 1972, S. 149-153.

38 William Hogarth: „Paul before Felix“, 1747/48, London, Lincoln’s Inn - vgl. u. a. Paulson, 1971 (wie Anm. 29), Bd. 2, S. 51-54; Werner Busch, Das sentimentalische Bild. Die Krise der Kunst im 18. Jahrhundert und die Geburt der Moderne, München 1993, S. 31-36. Bezeichnenderweise publizierte Hogarth eine Graphik mit dem Titel „Paul before Felix Burlesqued“, laut Bildunterschrift „Design’d and scratch’d 


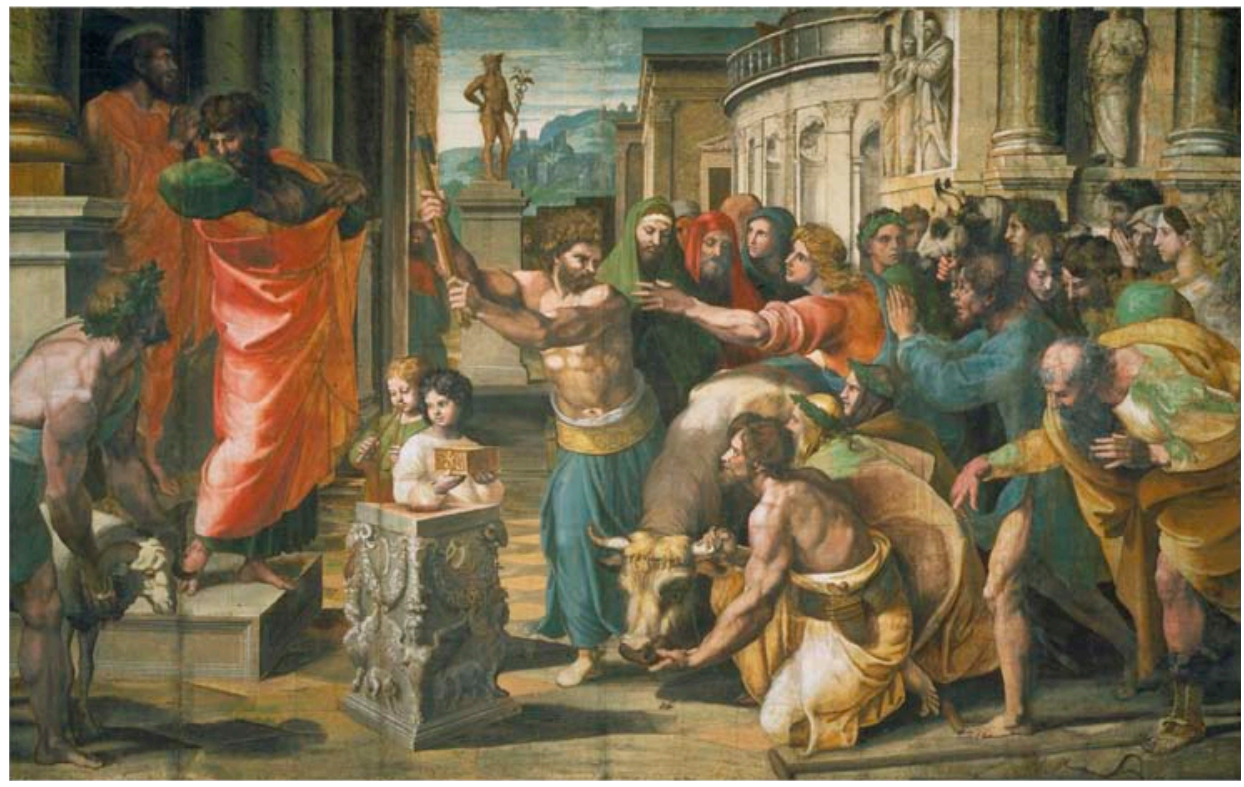

Abb. 4: Raffael: Das Opfer zu Lystra, 1514/15, schwarze Kreide und Tempera auf mehreren übereinandergeleimten und auf Leinwand kaschierten Papierbögen, 342 x $442 \mathrm{~cm}$, London, V\&A Images Victoria and Albert Museum

folglose - Bestreben muss durchaus ernst genommen werden. Einen wichtigen Beleg für sein Kunstverständnis bietet die Graphik „Characters and Caricatures“, die 1743 als Subskriptionsblatt für die Serie „Marriage à la Mode“ gedruckt wurde (Abb. 5). ${ }^{39}$ Im unteren Bereich kontrastiert sie - durch einen Riss getrennt - anhand älterer Beispiele aus der Kunstgeschichte Charakterdarstellungen mit karikaturistisch überzeichneten Kopfdarstellungen nach Annibale Carracci, Pier Leone Ghezzi und Leonardo da Vinci. ${ }^{40}$ Die - positiv konnotierten - Charakterköpfe stammen von Raffael. Einer davon ist aus dem Karton „Das Opfer zu Lystra“ übernommen, den Hogarth in „Strolling actresses“ parodiert. Betrachtet man das Blatt insgesamt, so bietet es ein Panorama dessen, was Hogarth physiognomisch und pathognomisch in seinem Werk ausgebreitet hat. Hogarth selbst inszeniert sich also zumindest hier als Charakterisierer und nicht als Karikaturist, wobei er sich unmissverständlich auf Raffael bezieht. Dass er ihn vorher parodiert hat, ändert ebensowenig an diesem Selbstverständnis wie

in the true Dutch taste“, um die Distanz zwischen der Höhenlage seines Historiengemäldes und einem niederen Stil in der Art holländischer Malerei zu demonstrieren - vgl. Werner Busch, 1993 (wie Anm. 38), S. 35f.; Paulson, 1970 (wie Anm. 4), Bd. 1, S. 215 f.

39 William Hogarth, Characters and Caricatures, 1743, Radierung, 206 × 200 mm; vgl. Paulson, 1970 (wie Anm. 4), Bd. 1, S. 188f.

40 Siehe die Beschriftung des Blattes - vgl. ebd. 


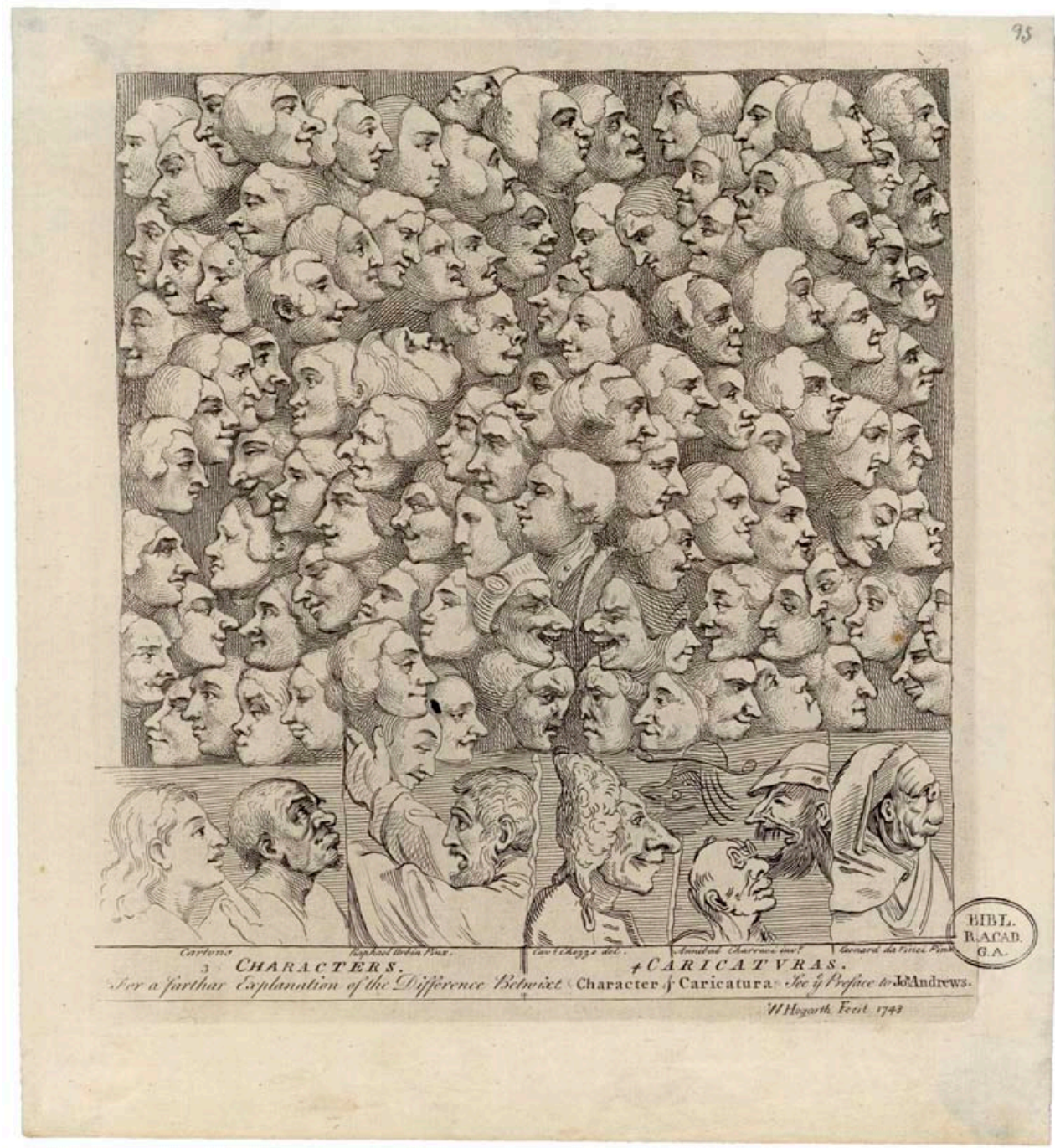

Abb. 5: William Hogarth: Characters and Caricatures, 1743, Radierung, 231 x 206 mm, Göttingen, Staats- und Universitätsbibliothek, gr. $2^{\circ}$ Art. plast. VI, 25 Rara: Abb. Nr. 21; mit freundlicher Unterstützung der Göttinger Staats- und Universitätsbibliothek 
die Frage, ob ihm die Umsetzung dieser Differenzierung - d.h. die Wahrung eines Abstands zur Karikatur - immer gelungen ist. ${ }^{41}$

Als Charakterisierer reiht sich Hogarth bei aller Spezifik, mit der er etwa auch gesellschaftskritische Themen umgesetzt hat, in eine breite und facettenreiche künstlerische und kunsttheoretische Tradition ein, welche von der Frühen Neuzeit bis ins 19. Jahrhundert hinein reicht. Es würde den Rahmen dieses Beitrags sprengen, hier eine angemessene Verortung des Künstlers zu bieten. Angedeutet sei hier nur, dass sich die Fähigkeit, Personen physiognomisch und pathognomisch zu charakterisieren, gerade für die Hochkunst der Historie mit dem Anspruch verband, Ausdrucksgehalte auf differenzierte Weise visualisieren zu können und damit etwas zu leisten, was den Sprachkünsten ebenbürtig sei.

Dass Georg Christoph Lichtenberg genau diese Fähigkeit bei Hogarth sah und besonders schätzte, zeigt sich an einer Ebene des Medientransfers, die bislang noch nicht zur Sprache gekommen ist, weil sie bei den "Strolling Actresses“ nicht vorkommt: Die kleinformatigen, von Ernst Ludwig Riepenhausen ab 1785 gestochenen Illustrationen zum „Göttinger Taschen Calender" konzentrieren sich nämlich nur auf die Köpfe der Personen, auf welche mit Zahlenverweisen im Text eingegangen wird.

Es ist wohl in erster Linie diese Charakterisierungskunst, der William Hogarth das große Lob Lichtenbergs zu verdanken hat: Den allgemeinen Beyfall hat er, wie große Schriftsteller den ihrigen, der Darstellung allgemeiner menschlicher Natur, und der Sprache zu danken, die man in Lissabon so gut versteht als in Moskau. ${ }^{42}$ Genau darin bestand das Ziel des Charakterisierens: In der Befähigkeit zum idealerweise allgemein-menschlich wirksamen Darstellen. Dass Hogarths Werk gleichwohl immer erklärungsbedürftig blieb und bleiben wird, war Lichtenberg nichtsdestoweniger klar und bildete sicher einen Hauptanlass für seine eindrucksvollen Leistungen im englisch-deutschen Kultur- und Medientransfer. Letztlich erscheint dieses Eingeständnis ehrlicher als das wohl von vornherein utopische Ziel einer unmittelbar und voraussetzungslos wirksamen Kunst. So braucht also die Kunst die Literatur, aber eben auch die Kunstgeschichte.

41 Vgl. Arburg, 1998 (wie Anm. 7), S. $188 f$.

42 Promies, 1999 (wie Anm. 1), S. 7. Vgl. auch Arburg, 1998 (wie Anm. 7), S. 195-210. 